Dies ist ein Preprint $(\mathrm{cc})$ EY-NC-ND. Die endgültige Publikationsfassung erscheint beim Verlag De Gruyter unter https://www.degruyter.com/view/j/bfup

Schwerpunkt Forschungsdaten

AR 3268

Ulrike Wuttke, Claus Spiecker und Heike Neuroth

\title{
PARTHENOS - Eine digitale Forschungsinfrastruktur für die Geistes- und Kulturwissenschaften
}

Zusammenfassung: Das EU-geförderte Projekt PARTHENOS steht für „Pooling Activities, Resources and Tools for Heritage E-research Networking, Optimization and Synergies" und arbeitet an der Verbesserung der Nutzung von digitalen Forschungsinfrastrukturen in den Geistes- und Kulturwissenschaften. Der Artikel stellt die Projekt-Ergebnisse der letzten drei Jahre, die sich auf eine technische und semantische Harmonisierung der verschiedenen bereits existierenden digitalen Forschungsinfrastrukturen wie z.B. DARIAH, CLARIN etc. konzentrieren, vor. Darüber hinaus hat das Projekt zum Ziel, durch spezifische Trainings- und Schulungsmaßnahmen weitere Forschende der EU an die Digital Humanities, deren Methoden und digitalen Dienste heranzuführen. Ein besonderer Fokus liegt dabei auch auf der Sensibilisierung für die Themen Open Science, FAIR und Forschungsdatenmanagement.

Schlüsselwörter: : Digital Humanities; Digitales Kulturerbe; Digitale Forschungsinfrastruktur

\section{PARTHENOS - A Digital Research Infrastructure in the Humanities and Cultural Sciences}

Abstract: The EU-funded PARTHENOS project stands for "Pooling Activities, Resources and Tools for Heritage E-research Networking, Optimization and Synergies" and works on improving the use of digital research infrastructures in the humanities and cultural sciences. The article presents the project results of the last three years, which focus on a technical and semantic harmonization of the different already existing digital research infrastructures such as DARIAH, CLARIN, etc. Furthermore, the project aims at introducing further researchers working in the EU to Digital Humanities, their methods and digital services through specific training and education measures. A special focus is also on raising awareness of the issues of Open Science, FAIR and research data management.

Keywords: Digital humanities; eHeritage, digital research infrastructure 
Preprints der Zeitschrift BIBLIOTHEK - Forschung und Praxis, 2019, AR 324 Wuttke, Spiecker und

\section{Neuroth}

Dies ist ein Preprint $(\mathrm{cc})$ EY-NC-ND. Die endgültige Publikationsfassung erscheint beim Verlag De Gruyter unter https://www.degruyter.com/view/j/bfup

\section{$1 \quad$ Motivation und Historie}

Die Forschung in den Bereichen Geisteswissenschaften und Kulturerbe unterliegt in den letzten Jahrzehnten einer rapiden digitalen Transformation (digital turn), insbesondere in den Bereichen, die als e-Humanities (bzw. Digital Humanities) und e-Heritage (bzw. Digital Heritage Science) bezeichnet werden. Die zunehmende Orientierung in Richtung datenbasierter Methoden und Fragestellungen verändert die Art und Weise, wie die Vergangenheit erforscht wird. Sie ermöglicht innovative Formen der Auseinandersetzung mit historischen Objekten und beeinflusst den gesamten Forschungs- und Publikationsprozess. Sie erfordert von den Wissenschaftlerinnen ${ }^{1}$ die Kenntnisnahme und kritische Auseinandersetzung mit den Möglichkeiten des vermehrten Einsatzes von digitalen Werkzeugen, Methoden und Technologien.

Computergestützte Analyseansätze können neue Perspektiven in traditionellen Forschungsbereichen sowie neue Forschungsbereiche durch Forschungsfragen, die vorher so nicht möglich waren, eröffnen. Die hierdurch entstehende Fülle digitaler Daten, Werkzeuge und Methoden stellt den Wissenschaftsbetrieb vor große Herausforderungen, die bisher nur unzureichend gelöst sind. Dies betrifft insbesondere die Entwicklung von übergreifenden und nachhaltigen Strategien bezüglich der Erzeugung, der Bewahrung und des Umgangs mit den digitalen Daten, sowohl im quantitativen als auch im qualitativen Sinn. Es müssen Mittel und Wege gefunden werden, um digitale Daten und Werkzeuge so zu bewahren und zu organisieren, dass ein internationaler und interdisziplinärer Austausch gewährleistet ist. Nur so wird es in Zukunft gelingen, gemeinsam Forschungsfragen in einer neuen Kultur der Zusammenarbeit zu entwickeln und zu beantworten sowie neue Publikationsund Gratifikationsstrukturen zu etablieren.

Bei der Entwicklung digitaler Methoden und Werkzeuge, der Erhebung von Daten sowie der Bereitstellung digitaler Forschungsprodukte für die Geisteswissenschaften und den Kulturerbebereich kommt sogenannten digitalen Forschungsinfrastrukturen bzw. der „Nationalen Forschungsdateninfrastruktur" (NFDI) ${ }^{2}$ eine zunehmend wichtige Rolle zu. Diese Entwicklung wird auch als infrastructural turn bezeichnet. Der Begriff digitale Forschungsinfrastruktur umfaßt im Kontext des vorliegenden Beitrags Maßnahmen und Einrichtungen, die den Einsatz von digitalen Methoden und Werkzeugen in allen Phasen des Forschungsprozesses unterstützen: von der Recherche über die Analyse bis hin zur Archivierung und Bereitstellung bzw. Publikation. Digitale Forschungsinfrastrukturen sind in gewissem Sinn die digitalen Pendants von „analogen“

\footnotetext{
${ }^{1}$ Aus Gründen besserer Lesbarkeit wird nur das Femininum verwendet. Selbstverständlich sind Personen jeden Geschlechts angesprochen.

2 Vgl. z.B. Richter (2018).
} 
Preprints der Zeitschrift BIBLIOTHEK - Forschung und Praxis, 2019, AR 324 Wuttke, Spiecker und

\section{Neuroth}

Dies ist ein Preprint $(\boldsymbol{C c})$ BY-NC-ND. Die endgültige Publikationsfassung erscheint beim Verlag De Gruyter unter https://www.degruyter.com/view/j/bfup

Forschungsinfrastrukturen wie Bibliotheken, Archiven, Museen oder von Weltraumteleskopen, um

ein Beispiel aus den Naturwissenschaften herauszugreifen. Seit langem übernehmen

Kulturerbeeinrichtungen wie Bibliotheken und Archive die Verantwortung dafür, Quellen für die Forschung zu identifizieren, zu ordnen, zu erhalten und zugänglich zu machen. ${ }^{3}$ Diese Aufgaben stellen sich auch im digitalen Bereich und bei ihrer Erfüllung entstehen ähnliche und neue Herausforderungen. Nicht zuletzt aus diesem Grund werden Aufbau und Verstetigung digitaler Forschungsinfrastrukturen in den Geisteswissenschaften unter starker Beteiligung von Kulturerbeeinrichtungen vorangetrieben.

Inzwischen existieren im Europäischen Forschungsraum (bzw. European Research Area, kurz ERA ${ }^{4}$ ) verschiedene bedarfsgetriebene digitale Forschungsinfrastrukturen für die Geistes- und Sozialwissenschaften und den Kulturerbebereich, die disziplinär oder interdisziplinär sowie national und international orientiert sein können. ${ }^{5}$ In dieser ausdifferenzierten Landschaft kommt vor allem denjenigen Forschungsinfrastrukturen eine tragende Rolle zu, die auf der nationalen Roadmap für Forschungsinfrastrukturen ${ }^{6}$ bzw. der europäischen Roadmap für Forschungsinfrastrukturen (ESFRI 2016) vertreten sind. Auf europäischer Ebene sind in diesem Zusammenhang die ERICs ${ }^{7}$ CLARIN ERIC und DARIAH-EU ERIC zu nennen. ${ }^{8}$ Auf dem Weg zu einer Akzeptanz in der Breite der von der Europäischen Kommission priorisierten Open Science Werte und FAIR-Prinzipien sowie der Realisierung der European Open Science Cloud (EOSC) spielen digitale Forschungsinfrastrukturen für die von ihnen bedienten Fachwissenschaften und Fach-Communities eine wichtige Rolle. ${ }^{9}$

Für die Wissenschaft ist die oben beschriebene ausdifferenzierte Infrastrukturlandschaft einerseits förderlich, da keine Infrastruktur den Bedürfnissen aller Forschenden gerecht werden kann, andererseits besteht die Gefahr der Fragmentierung. Es ist daher zwingend notwendig, dass die verschiedenen Stakeholder enger zusammenarbeiten, um optimale Bedingungen für Open Science und digitale Nachhaltigkeit zu schaffen, insbesondere durch die Vermeidung redundanter Entwicklungen bzw. der Entwicklung von Insellösungen. Hierauf beruht der Grundgedanke des von

\footnotetext{
${ }^{3}$ Siehe European Science Foundation (2011) 2.

${ }^{4}$ http://ec.europa.eu/research/era/index_en.htm.

${ }^{5}$ Siehe European Strategy Forum on Research Infrastructures (2016).

${ }^{6}$ https://www.bmbf.de/de/roadmap-fuer-forschungsinfrastrukturen-541.html.

7 ERIC steht für European Research Infrastructure Consortium https://ec.europa.eu/research/infrastructures/index.cfm?pg=eric.

8 Zu DARIAH-DE siehe Blümm, Neuroth, Schmunk (2016), zu CLARIN-D siehe Hinrichs, Trippel (2017).

${ }^{9}$ Siehe EOSC Pilot (2017).
} 
Preprints der Zeitschrift BIBLIOTHEK - Forschung und Praxis, 2019, AR 324 Wuttke, Spiecker und Neuroth

Dies ist ein Preprint $(\boldsymbol{C c})$ BY-NC-ND. Die endgültige Publikationsfassung erscheint beim Verlag De Gruyter unter https://www.degruyter.com/view/j/bfup

der Europäischen Kommission innerhalb des Rahmenprogramms Horizont $2020^{10}$ geförderten Projekts PARTHENOS ${ }^{11}$.

PARTHENOS ist ein Konsortium der wichtigsten europäischen Forschungsinfrastrukturen und Initiativen aus den Bereichen Linguistik, digitale Geisteswissenschaften, Geschichte, Kulturerbe und Archäologie mit dem erklärten Ziel, zwischen den einzelnen eng verwandten Fach-Communities Brücken zu bauen, um die Harmonisierung von Strategien, die Verbesserung der Datenqualität und die Erhöhung der Interoperabilität voranzutreiben. Wichtige Arbeitsbereiche von PARTHENOS sind unter anderem die Harmonisierung von Definitionen im Bereich Langzeitarchivierung und Intellectual Property Management sowie deren Umsetzung, die Entwicklung gemeinsamer Standards, Dienstleistungen und Methoden für die interdisziplinäre Nutzung und Nachnutzung von Daten sowie Aus- und Weiterbildung. Ein weiterer Arbeitsschwerpunkt sind Strategie-, Lobby- und Trainingsmaßnahmen bezüglich der FAIR-Prinzipien (FAIR steht für Findable, Accessible, Interoperable, Reusable ${ }^{12}$ und der Open Science Werte, mit dem Ziel ihrer breiteren Akzeptanz in den von PARTHENOS vertretenen geistes- und kulturwissenschaftlichen Fach-Communities und mit dem gemeinsamen Ziel, die Realisierung der EOSC zu unterstützen. ${ }^{13}$ Einige der von PARTHENOS entwickelten beispielhaften, integrativen Lösungsansätze werden im Folgenden detailliert vorgestellt.

\section{PARTHENOS - Kurzüberblick}

Der Name PARTHENOS - ein Akronym für Pooling Activities, Resources and Tools for Heritage Eresearch Networking, Optimization and Synergies - ist inspiriert von Athena Parthenos, der griechischen Göttin der Weisheit, Inspiration und Zivilisation. Im Mittelpunkt der Projektarbeit steht die Stärkung der Zusammenarbeit in den digital arbeitenden geistes- und kulturwissenschaftlichen Disziplinen. Der Projektantrag beantwortete die Ausschreibung INFRADEV-4-2014/2015 (Implementation and operation of cross-cutting services and solutions for clusters and other relevant research infrastructures initiatives) des Horizont 2020 Rahmenprogramms der Europäischen Kommission. ${ }^{14}$ Die Projektlaufzeit (48 Monate) startete im April 2015. Sie wurde unlängst kostenneutral um weitere sechs Monate verlängert, um den Projektpartnern die Möglichkeit zu

\footnotetext{
10 https://www.horizont2020.de.

$11 \mathrm{http}: / /$ www.parthenos-project.eu.

12 Siehe Wilkinson et al. (2016).

${ }^{13}$ Siehe PARTHENOS (2017).

14 PARTHENOS erhält finanzielle Unterstützung durch die Europäische Kommission unter der Horizont 2020 Fördernummer 654119.
} 
Preprints der Zeitschrift BIBLIOTHEK - Forschung und Praxis, 2019, AR 324 Wuttke, Spiecker und

\section{Neuroth}

Dies ist ein Preprint (c) BY-NC-ND. Die endgültige Publikationsfassung erscheint beim Verlag De Gruyter unter https://www.degruyter.com/view/j/bfup

geben, die Projektergebnisse intensiv in den Fach-Communities zu bewerben bzw. in Aus- und

Weiterbildungsmaßnahmen einfließen zu lassen.

Die Konsortialleitung und Koordination des europäischen Forschungsverbunds PARTHENOS liegt bei PIN s.c.r.I. ${ }^{15}$ (Italien). Das Konsortium besteht aus 16 Partnern aus neun Ländern (Italien, Frankreich, Griechenland, Niederlande, Irland, Vereinigtes Königreich, Spanien, Österreich und Deutschland). Es umfasst neben den beiden geisteswissenschaftlichen ERICs CLARIN und DARIAH-EU unter anderem universitäre und außeruniversitäre Forschungseinrichtungen wie Universitäten (PIN s.c.r.I., King's College London ${ }^{16}$, Trinity College Dublin ${ }^{17}$ ), Rechenzentren und nationale Forschungszentren $\left(\right.$ INRIA $^{18}, \mathrm{CNRS}^{19}{ }^{19}$ Huma-Num $\left.{ }^{20}, \mathrm{CSIC}^{21}, \mathrm{SISMEL}^{22}, \mathrm{FORTH}^{23}, \mathrm{CNR}^{24}\right)$, Akademien der Wissenschaften $\left(K_{N A W}{ }^{25}, O_{A W}{ }^{26}\right.$, Akademie von Athen $\left.{ }^{27}\right)$, die Fachhochschule Potsdam ${ }^{28}$ und die nationalbibliographische Forschungseinrichtung MIBACT-ICCU $29 .{ }^{30}$ Alle PARTHENOS-Projektpartner sind bzw. waren in verschiedenen Konstellationen an bedeutenden nationalen und internationalen geistes- und kulturwissenschaftlichen Forschungsinfrastrukturen wie E-RIHS ${ }^{31}, \mathrm{EHRI}^{32}$, Ariadne ${ }^{33}$, CENDARI $^{34}$ oder Isidore ${ }^{35}$ beteiligt, um nur einige Beispiele zu nennen. Dies erhöht maßgeblich die Einbindung und Akzeptanz der verschiedenen, digital arbeitenden geistes- und kulturwissenschaftlichen Fach-Communities. Darüber hinaus arbeitet PARTHENOS eng mit anderen nationalen und internationalen Initiativen wie zum Beispiel European ${ }^{36}$ zusammen und präsentiert

\footnotetext{
15 http://www.poloprato.unifi.it/it/home-polo.html?no_cache=1.

16 https://www.kcl.ac.uk/.

17 https://www.tcd.ie/.

18 https://www.inria.fr/.

19 https://www.cnrs.fr/.

20 https://www.huma-num.fr/.

${ }^{21}$ https://www.csic.es/.

22 https://www.sismel.it/.

${ }^{23}$ https://www.forth.gr/.

24 https://www.cnr.it/en.

${ }^{25}$ https://www.knaw.nl/.

26 https://www.oeaw.ac.at/.

${ }^{27}$ www.academyofathens.gr/.

28 https://www.fh-potsdam.de/.

29 https://www.beniculturali.it/mibac/opencms/MiBAC/sito-MiBAC/Luogo/MibacUnif/Luoghi-dellaCultura/visualizza_asset.html id=167208\&pagename $=174560$.

${ }^{30}$ Für eine Übersicht des Konsortiums siehe: http://www.parthenos-project.eu/consortium.

31 https://www.e-rihs.eu/.

32 https://www.ehri-project.eu/.

${ }^{33}$ https://www.ariadne-infrastructure.eu/.

${ }^{34}$ https://www.cendari.eu/.

35 https://isidore.science/.

${ }^{36}$ https://www.europeana.eu/portal/en.
} 
Preprints der Zeitschrift BIBLIOTHEK - Forschung und Praxis, 2019, AR 324 Wuttke, Spiecker und Neuroth

Dies ist ein Preprint $(\boldsymbol{c c )}$ EY-NC-ND. Die endgültige Publikationsfassung erscheint beim Verlag De Gruyter unter https://www.degruyter.com/view/i/bfup

Ergebnisse den verschiedenen Nutzer-Communities während nationaler und internationaler

Konferenzen und Tagungen sowie in Workshops des Projekts. ${ }^{37}$

\section{Die Kernbereiche von PARTHENOS}

Der Schwerpunkt der Arbeit von PARTHENOS liegt auf den fünf Komponenten:

- Harmonisierung von Richtlinien und Definitionen für digitale Technologien und Daten,

- Harmonisierung von (technischen) Standards,

- Entwicklung einer semantisch interoperablen technischen Infrastruktur für Dienstleistungen und Werkzeuge,

- Aufbau von Aus- und Weiterbildungsangeboten sowie

- Aktivitäten und innovativen Dienstleistungen im Bereich wissenschaftliche Kommunikation.

Hierbei stehen übergreifende fachwissenschaftliche Fragestellungen und Erkenntnisinteressen besonders im Mittelpunkt.

Das PARTHENOS-Projekt ist seinen Zielen entsprechend in acht Arbeitspakete (work packages) mit jeweils verschiedenen Einzelaufgaben (tasks) aufgeteilt. Der PARTHENOS-Projektpartner Fachhochschule Potsdam ist als Taskleader oder Taskmitglied an fünf Arbeitspaketen beteiligt. Im Folgenden werden die Arbeitspakete 2-8 vorgestellt. Dabei wird einerseits kurz auf grundlegende Ergebnisse der Arbeit der einzelnen Arbeitspakete eingegangen sowie einige Ergebnisse, an denen die Fachhochschule Potsdam maßgeblich beteiligt war, ausführlicher dargestellt. Arbeitspaket 1 (Project Coordination and Management) ist verantwortlich für die allgemeine Steuerung des Projekts und wird daher nicht detailliert vorgestellt.

\subsection{Arbeitspaket 2 "Community Involvement and Requirements“}

Zentral für die Arbeit von PARTHENOS sind die Ermittlung und der permanente Abgleich der Projektergebnisse mit den Anforderungen der Fach-Communities. Deshalb wurde in dem Projektaufbau und dem Arbeitsablauf eine permanente Feedbackschleife im Sinne der agilen Softwareentwicklung über das hierfür zentrale Arbeitspaket 2 eingebaut.

Die Arbeit der Arbeitspakete 3-8 fußt auf den Ergebnissen der umfassenden Analyse der Nutzeranforderungen durch die Mitglieder von Arbeitspaket 2 in der Projekteingangsphase, d.h. der Feinabstimmung der im Projektantrag formulierten grundsätzlichen Zielstellungen der einzelnen Arbeitspakete. ${ }^{38}$ Als wichtigste Nutzergruppen des PARTHENOS-Projekts wurden

\footnotetext{
37 Die Aktivitäten des Projekts werden über die Webseite (http://www.parthenos-project.eu), verschiedene Social Media Kanäle (wie Facebook oder Twitter) sowie einen Newsletter angekündigt.

38 Siehe Drude et al. (2016).
} 
Preprints der Zeitschrift BIBLIOTHEK - Forschung und Praxis, 2019, AR 324 Wuttke, Spiecker und Neuroth

Dies ist ein Preprint $(\mathrm{cc})$ EY-NC-ND. Die endgültige Publikationsfassung erscheint beim Verlag De Gruyter unter https://www.degruyter.com/view/i/bfup

Wissenschaftlerinnen der Disziplinen Geschichte, Philologien (sprach- und textbasierte Disziplinen), Archäologie sowie Praktikerinnen aus dem Kulturerbebereich wie Bibliothekarinnen, Archivarinnen, Museologinnen, Datenkuratorinnen und -kuratoren identifiziert. ${ }^{39}$ Die Nutzeranforderungen wurden in der Projekteingangsphase weitgehend auf der Grundlage publizierter Ergebnisse relevanter Vorgängerprojekte ermittelt und dann durch den direkten Input der PARTHENOS-Partner ergänzt. Die Ergebnisse wurden zu detaillierten Use Cases als Richtlinien für die Arbeit der einzelnen Arbeitspakete während der Projektlaufzeit aufbereitet (Drude et al. 2016). Des Weiteren sind im Arbeitsplan kontinuierlich Feedbackschleifen für die Ergebnisse der einzelnen Arbeitspakete unter Leitung von Arbeitspaket 2 vorgesehen, zu denen teilweise Einzelberichte veröffentlicht werden. ${ }^{40}$ Diese Methode stellt die direkte Einbindung der Fach-Communities in die Entwicklungen des Projekts sicher (Bottom-Up-Approach), erhöht die Innovationskraft und dient der Qualitätssicherung.

\subsection{Arbeitspaket 3 „Common Policies and Implementation Strategies"}

Im Mittelpunkt dieses Arbeitspakets stehen Richtlinien für das Datenmanagement und ihre Umsetzung in die Praxis. Auf der Basis der durch Arbeitspaket 2 ermittelten Nutzeranforderungen wurde von Arbeitspaket 3 der Report on Guidelines for Common Policies Implementation ${ }^{41}$ erstellt. Den Schwerpunkt dieses Berichts bilden die Beurteilung der Qualität von Daten, Metadaten und Repositorien sowie die Umsetzung von Richtlinien in den Bereichen Datenmanagement, Intellectual Property Rights (IPR), Open Data und Open Access unter besonderer Berücksichtigung der FAIRPrinzipien ${ }^{42}$. Auf dieser Basis werden Leitsätze und Prinzipien für die Erleichterung der Umsetzung in die Praxis entwickelt. ${ }^{43}$ Parallel dazu wird die Vorlage für den PARTHENOS Data Management Plan für die digitalen Geisteswissenschaften entwickelt. Er soll unter Berücksichtigung der FAIR-Prinzipien den Anforderungen unterschiedlicher Nutzergruppen wie institutionellen Repositorien, Forschungsprojekten und Wissenschaftlerinnen und ihre jeweilige Sicht auf die Datenqualität gerecht werden. Um den Nutzerinnen das Ausfüllen des Datenmanagementplans zu erleichtern, werden zur Orientierung disziplinspezifische Erläuterungen bereitgestellt.

\subsection{Arbeitspaket 4 „Standardization“}

Die Forschung in Arbeitspaket 4 konzentriert sich auf den Bereich Standardisierung in den Geistesund Kulturwissenschaften. Als wichtigste Handlungsfelder wurden durch PARTHENOS identifiziert:

\footnotetext{
39 Siehe Drude et al. (2016) 1.

${ }^{40}$ Siehe u.a. Oltersdorf et al. (2016), Krauwer et al. (2017). Weitere Evaluationen und Berichte sind in Arbeit. Alle offiziellen PARTHENOS Berichte (Deliverables) sind auf der Projektwebseite zu finden: http://www.parthenosproject.eu/resources/projects-deliverables.

${ }^{41}$ Siehe Hollander et al. (2017).

42 https://www.go-fair.org/fair-principles/.

${ }^{43}$ Eine eigenständige Veröffentlichung zu den Empfehlungen ist für Anfang 2019 geplant.
} 
Preprints der Zeitschrift BIBLIOTHEK - Forschung und Praxis, 2019, AR 324 Wuttke, Spiecker und

\section{Neuroth}

Dies ist ein Preprint $(\mathrm{Cc})$ EY-NC-ND. Die endgültige Publikationsfassung erscheint beim Verlag De Gruyter unter https://www.degruyter.com/view/i/bfup

- "Community Standards to document primary data and sources" (zum Beispiel TEl ${ }^{44}$, CIDOC-CRM ${ }^{45}, \mathrm{CMDI}^{46}$, Alto-XML ${ }^{47}, \mathrm{MEI}^{48}$ ),

- „Reference Resources“ (zum Beispiel Normdaten und kontrollierte Vokabularsysteme) und

- "Protocols and procedures for the Cultural Heritage domains“. ${ }^{49}$

Diese drei Bereiche befinden sich in verschiedenen Stadien der Standardisierung, wodurch sich für die Aktivitäten von Arbeitspaket 4 unterschiedliche Stoßrichtungen bedingen:

- Förderung und Bewusstseinsbildung in denjenigen Fällen, in denen bereits tragfähige Standards existieren,

- Weiterentwicklung derjenigen Standards, die sich noch in der Definitionsphase befinden,

- Vorarbeiten leisten in denjenigen Domänen, in denen momentan keine Standards existieren (wie zum Beispiel für digitale 3-D-Objekte). ${ }^{50}$

Das wichtigste Produkt von Arbeitspaket 4 ist das sogenannte „Standardization Survival Kit“ (kurz SSK). ${ }^{51}$ Das SSK ist eine umfassende Online-Dokumentation mit dem Ziel, den Nutzerinnen grundlegende Informationen und Dokumentationen zu relevanten Standards in einer breiten Spanne der digital arbeitenden Geistes- und Kulturwissenschaften bereitzustellen. Hierfür wurden im Rahmen des SSK grundlegende Forschungsszenarien entsprechend des jeweiligen aktuellen Wissensstandes der Fach-Communities entlang der Phasen des Forschungszyklus annotiert. Das SSK soll insbesondere fortgeschrittenen Nutzerinnen einen zentralen Einstiegspunkt bieten, der es ihnen ermöglicht, sich je nach individuellem Informationsbedürfnis anhand autorisierter Materialien und Referenzen mit dem jeweiligen Thema auseinanderzusetzen. Es kann aber auch in der Lehre verwendet werden. Des Weiteren erarbeitet Arbeitspaket 4 in Zusammenarbeit mit den Arbeitspaketen 7 und 8 auch Kommunikations- und Trainingsmaterialien zur Bedeutung von Standards für den Forschungsprozess. Diese Materialien stellen auf spielerische Art und Weise die essentielle Rolle der Verwendung von Standards für die breite Nachnutzung von Forschungsdaten und -ergebnissen dar. Bisher wurden u.a. ein Flyer erarbeitet, in dessen Mittelpunkt die Comic-

\footnotetext{
${ }^{44}$ https://www.tei-c.org/.

45 https://www.cidoc-crm.org/.

${ }^{46}$ https://www.clarin.eu/content/component-metadata.

47 https://www.loc.gov/standards/alto/.

48 https://music-encoding.org/.

${ }^{49}$ Zitiert nach Romary et al. (2017) 1.

${ }^{50}$ Siehe Romary et al. (2017) 2.

${ }^{51}$ https://ssk-application. parthenos.d4science.org/ssk/\#/.
} 
Preprints der Zeitschrift BIBLIOTHEK - Forschung und Praxis, 2019, AR 324 Wuttke, Spiecker und Neuroth

Dies ist ein Preprint $(\mathrm{cc})$ EY-NC-ND. Die endgültige Publikationsfassung erscheint beim Verlag De Gruyter unter https://www.degruyter.com/view/i/bfup

Figuren Mork und Tork stehen sowie ein kurzer, auf den Comic-Charakteren des Flyers basierender Animationsfilm, der in mehreren Sprachen auf dem PARTHENOS Youtube Channel zu finden ist. ${ }^{52}$

\subsection{Arbeitspaket 5 „Interoperability and Semantics“ und Arbeitspaket 6 „Services and Tools"}

Im Mittelpunkt der Arbeitspakete 5 und 6 steht die technische Infrastruktur des gesamten Projekts. Auch diese beiden Arbeitspakete greifen auf die Vorarbeiten von Arbeitspaket 2 zurück, das die sehr heterogenen Anforderungen der unterschiedlichen Disziplinen zusammengetragen hat. Um die Interoperabilität, insbesondere bei der Integration verschiedensprachiger Quellen (wie Ontologien, Thesauri, Verzeichnisse, Berechtigungslisten) zu gewährleisten, war u.a. die Definition einer gemeinsamen semantischen Struktur ${ }^{53}$ für die Cloud-basierte Infrastruktur ${ }^{54}$ erforderlich. Eine Herausforderung stellte dabei das Mapping der unterschiedlichen Metadatenformate der am Projekt beteiligten Partner EHRI, ARIADNE und CLARIN dar, da diese für unterschiedliche Fach-Communities und Wissensgebiete wie zum Beispiel Holocaustforschung, Kulturerbe, Archäologie oder Linguistik entwickelt worden waren. ${ }^{55} \mathrm{Um}$ ein adäquates Mapping zu ermöglichen, wurde das PARTHENOS Entity Model entwickelt und stetig an die Anforderungen angepasst. ${ }^{56}$

Die digitale Infrastruktur des Projekts wird u.a. durch die Entwicklung und Integration der „Joint Resource Registry “57, ein umfassendes Verzeichnis der Ressourcen und Werkzeuge, konsequent zur einer PARTHENOS Virtuellen Forschungsumgebung (Virtual Research Environment) ausgebaut. Die Umsetzung der Anforderungen wurde durch Arbeitspaket 2 evaluiert und anhand des Feedbacks sind kontinuierlich Verbesserungen der technischen Infrastruktur beispielsweise in Hinblick auf die Integration von speziellen Anforderungen bisher nicht berücksichtigter Disziplinen durchgeführt worden.

\subsection{Arbeitspaket 7 „Skills, Professional Development and Advancement“}

Ein wichtiger Aspekt der Arbeit von PARTHENOS ist die Wissensvermittlung über die Rollen, Funktionen und Potentiale digitaler Forschungsinfrastrukturen im geistes- und kulturwissenschaftlichen Bereich durch Trainings- und Ausbildungsangebote. Die Trainingsangebote und -materialien werden von Arbeitspaket 7, insbesondere während der zweiten Phase in dem von der FHP geleiteten Task 7.2, in enger Kooperation mit allen PARTHENOS-Arbeitspaketen und

\footnotetext{
52 Die deutsche Version des Animationsfilms ist verfügbar unter https://youtu.be/ZfTHPbDTX1Y.

53 Siehe Bruseker, Doerr, Theodoridou (2017).

${ }^{54}$ Siehe Pagano et al. (2016) und Frosini et al. (2018).

55 Siehe z.B. Ďurčo, Lorenzini, Sugimoto (2018).

${ }^{56}$ Siehe Bruseker, Doerr, Theodoridou (2017).

${ }^{57}$ Siehe Alois et al. (2017).
} 
Preprints der Zeitschrift BIBLIOTHEK - Forschung und Praxis, 2019, AR 324 Wuttke, Spiecker und

\section{Neuroth}

Dies ist ein Preprint $(\boldsymbol{C c})$ BY-NC-ND. Die endgültige Publikationsfassung erscheint beim Verlag De Gruyter unter https://www.degruyter.com/view/j/bfup

Projektpartnern erarbeitet. ${ }^{58}$ Sie richten sich an Wissenschaftlerinnen sowie Praktikerinnen aus den

Geisteswissenschaften und aus dem Kulturerbebereich, aber auch an Entwicklerinnen und

Mitarbeiterinnen in Rechenzentren sowie an Entscheidungsträgerinnen, da die Analyse der

Nutzeranforderungen und Nutzergruppen in der Eingangsphase des Projekts gezeigt hat, dass

grundlegendes Wissen über die Rolle und Funktion von Forschungsinfrastrukturen für eine breitere

Nutzergruppe relevant ist. Bei der Erarbeitung der Lehrangebote und -materialien unterscheidet das PARTHENOS Training zwischen den grundlegenden didaktischen Zielen „Bewusstseinsbildung“ (awareness raising) und dem „Erwerb von Kompetenzen“ (skills building). Von einer Ausdifferenzierung bezüglich verschiedener professioneller Stadien (wie Graduierte oder Postdocs) wird weitgehend abgesehen, da diese meist wenig über die digitalen Kompetenzen aussagen. Es wird jedoch ein Unterschied zwischen Angeboten für Anfänger, Fortgeschrittene und Experten gemacht. Hervorzuheben ist die Erarbeitung eines speziell auf ein universitäres Curriculum ausgerichteten Piloten mit PARTHENOS Trainingsmaterialien am King's College London.

Auf Basis aller an PARTHENOS beteiligten Partner wurde projektübergreifend unter Koordination von Arbeitspaket 7 die PARTHENOS Training Suite ${ }^{59}$ entwickelt. Diese ist eine auf WordPress basierte Online-Plattform, die aus einer Reihe von Modulen besteht. Die Module fokussieren auf infrastrukturell breit angelegten Themengebieten wie „Introduction to Research Infrastructures", „Management Challenges in Research Infrastructures", und „Introduction to Collaborations in Research Infrastructures“. Die Plattform wird laufend weiterentwickelt und thematisch erweitert, um konsekutiv eine breite Spanne von infrastrukturell relevanten Anforderungen, Themengebieten und Wissensständen in den digitalen Geistes- und Kulturwissenschaften abzubilden. Zu den jüngsten Erweiterungen zählen das Modul „Open Up your Research and Data“ sowie zwei Teilmodule zum Thema „Impact“. Weitere Module, unter anderem zu Ontologien, Citizen Science und der Entwicklung von Forschungsfragen auf der Grundlage von durch digitale Forschungsinfrastrukturen bereitgestellten Werkzeugen, sind in Vorbereitung. Innerhalb der Training Suite ist eine unterschiedliche Breite an Lehr- und Lern-Materialien verfügbar wie beispielsweise Video-Vorträge, Erklärfilme, Präsentationsmaterialien, Übungen und Erläuterungen zu Grundprinzipien und Broschüren. Die bereitgestellten Materialien eignen sich für asynchrone Selbststudienzwecke, sie sind jedoch vor allem für die individuelle Weiterverwendung durch Lehrende im Rahmen eigener Lehrveranstaltung gedacht.

\footnotetext{
58 Siehe Edmond et al. (2016) und Spiecker et al. (2017).

59 http://training.parthenos-project.eu/.
} 
Preprints der Zeitschrift BIBLIOTHEK - Forschung und Praxis, 2019, AR 324 Wuttke, Spiecker und Neuroth

Dies ist ein Preprint $(\mathrm{cc})$ EY-NC-ND. Die endgültige Publikationsfassung erscheint beim Verlag De Gruyter unter https://www.degruyter.com/view/i/bfup

Der Schwerpunkt von PARTHENOS Training liegt auf der Entwicklung von unterschiedlichen Materialien für das Selbststudium sowie die Integration in die Lehre und umfasst daher auch unterschiedliche Veranstaltungstypen. So beteiligen sich an PARTHENOS affiliierte

Wissenschaftlerinnen an bekannten Konzepten wie Sommer- und Winterschulen sowie Methodenund Expertenworkshops oder halten Vorträge zu den didaktischen Zielen und Methoden für verschiedene Zielgruppen. Besonders hervorzuheben ist in diesem Zusammenhang die erfolgreiche Kooperation mit der Leipziger European Summer University in Digital Humanities (ESU). ${ }^{60}$

Während der Monate Februar und April 2018 hat PARTHENOS Training eine Reihe von fünf äußerst erfolgreichen Webinaren unter dem Titel „PARTHENOS eHumanities and eHeritage Webinar Series“ durchgeführt. ${ }^{61}$ Dieses von den Projektpartnern Fachhochschule Potsdam und Universität Leipzig gemeinsam entwickelte und in Zusammenarbeit mit namhaften Experten durchgeführte eLearningFormat bot die Gelegenheit, eine disziplinär und geografisch breit gefächerte Zielgruppe virtuell „live“ zu erreichen. Die Webinare waren thematisch entlang des Forschungszyklus verortet und boten aus infrastruktureller Sicht einen Einblick in bedeutende Themenbereiche der digitalen Geisteswissenschaften und des digitalen Kulturerbes. Der große Erfolg der Serie zeigt, dass sie einen Nerv getroffen hat. Alle Webinarmaterialien (wie Präsentationsfolien und Aufnahmen der Webinare) stehen zur Nachnutzung auf der PARTHENOS Training Suite zur Verfügung ${ }^{62}$ und eine Publikation zu den Erfahrungswerten ist geplant, um Projekten und Kulturerbeeinrichtungen eine Handreichung für eigene Aktivitäten in diesem Bereich zu bieten. ${ }^{63}$

\subsection{Arbeitspaket 8: "Communication, dissemination and outreach"}

Arbeitspaket 8 organisiert und koordiniert alle Kommunikations-, Informations- und

Marketingaktivitäten. Es bespielt verschiedene Kommunikationskanäle, um die Projektziele und Ergebnisse bei den unterschiedlichen Zielgruppen, z.B. Wissenschaftlerinnen,

Entscheidungsträgerinnen sowie der interessierten Öffentlichkeit bekannt zu machen. Innerhalb der ersten Monate wurde die PARTHENOS-Homepage als zentraler Einstiegspunkt erstellt. ${ }^{64}$ Sie dient als Hauptinformationskanal und informiert über alle Aktivitäten (wie Trainings, Workshops und Events), Projektergebnisse (u.a. Projektberichte und -produkte) und über die beteiligten und assoziierten Partner. Ein regelmäßig erscheinender Newsletter sowie Social Media-Aktivitäten (Twitter, Facebook etc.) informieren die Nutzerinnen zusätzlich über neue Entwicklungen. Die Online-Aktivitäten

\footnotetext{
$60 \mathrm{http}: / /$ www.culingtec.uni-leipzig.de/ESU_C_T/node/97.

${ }^{61} \mathrm{http} / / /$ www.parthenos-project.eu/successful-parthenos-ehumanities-and-eheritage-webinar-series-concluded/sowie Wuttke, Sierig, Neuroth (2018, June).

$62 \mathrm{http} / / /$ training.parthenos-project.eu/sample-page/ehumanities-eheritage-webinar-series.

63 Wuttke (in prep. 2019).

64 http://www.parthenos-project.eu.
} 
Preprints der Zeitschrift BIBLIOTHEK - Forschung und Praxis, 2019, AR 324 Wuttke, Spiecker und

\section{Neuroth}

Dies ist ein Preprint $(\boldsymbol{C c})$ BY-NC-ND. Die endgültige Publikationsfassung erscheint beim Verlag De Gruyter unter https://www.degruyter.com/view/j/bfup

werden durch die Entwicklung und Verbreitung von gedruckten Informationsmaterialien wie Flyern und Postern begleitet und unterstützt.

Ein wichtiges Anliegen von Arbeitspaket 8 ist es, Synergieeffekte durch Kooperationen auszuschöpfen und den Ausbau des Netzwerks voranzutreiben. Die Organisation von Veranstaltungen wie Konferenzen, Workshops und öffentlichen Präsentationen - vielfach in Kooperation mit anderen internationalen Initiativen - dient der Verbreitung der Projektergebnisse, der Stärkung der Zusammenarbeit der verschiedenen eher fragmentierten

Forschungsinfrastrukturen und führt oft zu weiteren gemeinsamen Aktivitäten in Forschung und Lehre sowie Veranstaltungen.

Neben den bereits beschriebenen Instrumenten für die Öffentlichkeitsarbeit werden in diesem Arbeitspaket auch neue Möglichkeiten im Hinblick auf eine Veränderung und Verbesserung der wissenschaftlichen Kommunikation geprüft. Ziel ist es, eine Verbindung zwischen etablierten Verfahren des wissenschaftlichen Austauschs, wie zum Beispiel Zeitschriften, und alternativen Verfahren im Social Media-Bereich, wie zum Beispiel Blogs und sozialen wissenschaftlichen Netzwerken wie ResearchGate ${ }^{65}$, Mendeley ${ }^{66}$ oder Academia ${ }^{67}$, zu schaffen. Hierzu wird von diesem Arbeitspaket zu Testzwecken der PARTHENOS Hub ${ }^{68}$ als innovative Publikations- und Interaktionsplattform entwickelt. Veränderliche Publikationen wie zum Beispiel Blogbeiträge oder Wikipedia-Artikel ${ }^{69}$ werden hierfür im PDF-Format ${ }^{70}$ dauerhaft in einem Repositorium gespeichert und mit Hilfe eines Persistent Identifier auf dem PARTHENOS Hub zusammen mit traditionellen Artikeln zu dem gleichen Thema in einer Ausgabe („Issue“) veröffentlicht. Die Verwendung von Hypothes.is ${ }^{71}$ ermöglicht eine direkte, fachwissenschaftliche Kommentierung der Inhalte. Hierdurch verbindet der PARTHENOS Hub die Vorteile beider Verfahren - Qualitätssicherung, Zitierbarkeit und wissenschaftliche Anerkennung einerseits und schnellen, direkten Austausch andererseits.

\footnotetext{
65 https://www.researchgate.net.

66 https://www.mendeley.com.

$67 \mathrm{https} / / / \mathrm{www}$. academia.edu.

$68 \mathrm{http}: / /$ www.parthenos-project.eu/portal/the-hub und Spiecker (2018).

69 https://de.wikipedia.org.

70 https://www.pdfa.org/.

${ }^{71}$ https://web.hypothes.is/.
} 
Preprints der Zeitschrift BIBLIOTHEK - Forschung und Praxis, 2019, AR 324 Wuttke, Spiecker und

\section{Neuroth}

Dies ist ein Preprint $(\boldsymbol{C c})$ BY-NC-ND. Die endgültige Publikationsfassung erscheint beim Verlag De Gruyter unter https://www.degruyter.com/view/j/bfup

\section{$4 \quad$ Fazit und Ausblick}

In Hinblick auf zukünftige Entwicklungen im Infrastrukturbereich wurde von PARTHENOS eine Umfrage zu den FAIR-Prinzipien und der zukünftigen EOSC durchgeführt. ${ }^{72}$ Auch wenn die Umfrage (ca. 100 Teilnehmerinnen und Teilnehmer) nicht als repräsentativ für alle in PARTHENOS vertretenen Fach-Communities angesehen werden kann, offenbarte sie doch signifikante Defizite hinsichtlich der Bekanntheit der FAIR-Prinzipien und des EOSC-Konzepts bzw. seit November 2018 geplanten Implementierung. Sie lieferte außerdem wertvolle Hinweise dafür, wie eine breite Akzeptanz dieser Schlüsselkonzepte in den von PARTHENOS bedienten Fach-Communities vorangetrieben werden kann. Die identifizierten Strategien reichen von kurzfristig umsetzbaren Instrumenten wie spezifischen Trainings- und Ausbildungsangeboten bis hin zu der Ableitung von langfristigen Umsetzungsszenarien. Hierzu zählen unter anderem beschleunigte Verfahren, um bereits verfügbare und qualitätsgesicherte digitale Dienste in eine Cloud-Umgebung zu integrieren, die weitere Notwendigkeit von Definitionen für gemeinsame Standards und die Entwicklung einfach anzuwendender Mechanismen, die die parallele Nutzung mehrerer Forschungsinfrastrukturen und deren Angebote sowie Werkzeuge erlaubt, unabhängig davon, welche Teil-Disziplin diese in erster Linie bedient.

Insgesamt liefert PARTHENOS einen wichtigen Beitrag im Bereich der Interdisziplinarität und Interoperabilität und hilft, die Fragmentierung von Forschungsinfrastrukturen in den Geistes- und Kulturwissenschaften zu überwinden. So wurde durch PARTHENOS die Kooperation zwischen den bereits existierenden digitalen Forschungsinfrastrukturen erhöht und damit über Disziplingrenzen hinweg der Austausch über Standards, sowohl technischer als auch semantischer Natur, deutlich angeregt. Dies sind beste Voraussetzungen für eine spätere Implementierung der FAIR Prinzipien, die gerade auf die Nachnutzung von digitalen Forschungsdaten abzielen. PARTHENOS ist es dabei gelungen, auch über Domaingrenzen hinweg Diskussionen und Entwicklungen anzustoßen, da nicht nur innerhalb der wissenschaftlichen Communities diskutiert wurde, sondern auch weitere relevante Player wie Vertreterinnen aus Rechenzentren, den Gedächtnisinstitutionen oder größeren Forschungsverbünden einbezogen werden konnten. Damit liefert PARTNENOS einen wichtigen Beitrag für die European Open Science Cloud (EOSC), die im November 2018 im Rahmen der österreichischen Ratspräsidentschaft von der Europäischen Kommission gelauncht wurde. Die Zielsetzung von EOSC ist es „to provide a safe environment for researchers to store, analyse and reuse data for research, innovation and educational purposes [...] across technologies, disciplines and

72 http://www.parthenos-project.eu/the-fair-principles-and-the-eosc-concept-in-parthenos-community sowie PARTHENOS (2017). 
Preprints der Zeitschrift BIBLIOTHEK - Forschung und Praxis, 2019, AR 324 Wuttke, Spiecker und

\section{Neuroth}

Dies ist ein Preprint (c) BY-NC-ND. Die endgültige Publikationsfassung erscheint beim Verlag De Gruyter unter https://www.degruyter.com/view/j/bfup

borders $^{\text {“73 }}$. Jede Anstrengung von Seiten der involvierten Stakeholder, im Vorfeld zu einer

Harmonisierung, Normierung (z.B. im Bereich kontrollierter Vokabularsysteme sowie Metadaten-

Formate) und Standardisierung im Umfeld digitaler Daten beizutragen, erleichtert naturgemäß die bestmögliche Annäherung bei der maximal möglichen Umsetzung der FAIR-Prinzipien. Damit soll nicht impliziert werden, dass eine hundertprozentige Umsetzung dieser Prinzipien möglich ist. Allerdings sollte es perspektivisch möglich sein, dass sich Fach-Communities, Forschungsverbünde bzw. potentielle Konsortien im Bereich "Nationaler Forschungsdateninfrastruktur (NFDI) ${ }^{474}$ in aussagekräftiger Form zu den FAIR-Prinzipien verhalten. Die Ankündigung der GWK zu der Förderung von NFDIs im Rahmen einer Bund-Länder-Vereinbarung zu Aufbau und Förderung ${ }^{75}$ benennt eindeutig die Kriterien: „Dazu muss das Datenmanagement standardisiert sein: Nach den sogenannten FAIR-Prinzipien sollen Forschungsdaten auffindbar, zugänglich, interoperabel und nachnutzbar sein." In diesem Kontext spielen die von PARTHENOS für die Geistes- und Kulturwissenschaften in die Wege geleiteten Diskussionen und Entwicklungen sowie konkreten Ergebnisse eine wichtige Grundvoraussetzung. PARTHENOS liefert jetzt bereits einen Beitrag zu der auch von der EOSC skizierten Idee „als digitaler, regional verteilter und vernetzter Wissensspeicher Forschungsdaten nachhaltig [zu] sichern und nutzbar [zu] machen. ${ }^{776}$ Dazu passen auch die beiden im November 2018 veröffentlichten Berichte der Europäischen Kommission:

- Prompting an EOSC in practice ${ }^{77}$ mit dem Ziel „to establishing the main Rules of Participation; also paying attention to issues as Governance and possible Business Models. The report analyses various aspects of how the EOSC can effectively interlink People, Data, Services and Training, Publications, Projects and Organisations and presents a set of detailed practical recommendations"

- Turning FAIR into reality ${ }^{78}$, ein Bericht, der "the broad range of changes required for the implementation of the FAIR data principles" beschreibt und direkt auf die Implementierung der FAIR Prinzipien zielt.

\section{Literaturverzeichnis}

Aloia, Nicola; Candela, Leonardo; Debole, Franca; Frosini, Luca; Lorenzini, Pagano, Matteo; Pasquale (2017). Design of the Joint Resource Registry. Veröffentlicht am 12.04.2017. Verfügbar unter

\footnotetext{
${ }^{73}$ Commission launches European Open Science Cloud, 23.November 2018. https://ec.europa.eu/info/news/commissionlaunches-european-open-science-cloud-2018-nov-23_en.

${ }^{74}$ Gemeinsame Wissenschaftskonferenz (GWK). Informationsinfrastrukturen/NFDI. https://www.gwkbonn.de/themen/weitere-arbeitsgebiete/informationsinfrastrukturen.

75 https://www.gwk-bonn.de/fileadmin/Redaktion/Dokumente/Papers/NFDI.pdf.

76 https://idw-online.de/de/news706198.

77 Report of the Commission 2nd High Level Expert Group on the European Open Science Cloud (EOSC 2nd HLEG), https://ec.europa.eu/info/publications/prompting-eosc-practice_en.

${ }^{78}$ Report of the Commission FAIR Data Expert Group (FAIR Data EG), https://ec.europa.eu/info/publications/turning-fair-reality_en.
} 


\section{Neuroth}

Dies ist ein Preprint (c) BY-NC-ND. Die endgültige Publikationsfassung erscheint beim Verlag De Gruyter unter https://www.degruyter.com/view/j/bfup

http://www.parthenos-

project.eu/Download/Deliverables/D5.2_Report_on_design_Joint_Resource_Registry.pdf.

Alliez, Pierre; Bergerot, Laurent; Bernard, Jean-François; Boust, Clotilde; Bruseker, George; Carboni, Nicola; Chayani, Mehdi; Dellepiane, Matteo; Dell'unto, Nicolo; Dutailly, Bruno; Gautier, Hélène; Guidi, Gabriele; Guillem, Anaïs; Joffres, Adeline; Laroche, Florent; Manuel, Adeline; Manzetti, Maria Cristina; Michel, Alain ; Pamart, Anthony; Ponce, Jean; Puren, Marie; Riondet, Charles; Rodriguez Echavarria, Karina; Romary, Laurent; Scopigno, Roberto; Tournon-Valiente, Sarah (2017): Digital 3D objects in the Arts and Humanities: challenges of creation, interoperability and preservation. PARTHENOS White Paper: A result of the PARTHENOS workshop held in Bordeaux at Maison des Sciences de l'Homme d'Aquitaine and at Archeovision Lab. (France), November 30th - December 2nd, 2016. DOI: hal-01526713v2. Verfügbar unter https://hal.inria.fr/hal$01526713 v 2$.

Blümm, Mirjam; Neuroth, Heike; Schmunk, Stefan (2016): DARIAH-DE - Architecture of Participation, In: BIBLIOTHEK - Forschung und Praxis, 40 (2), 165-71.

Bruseker, George; Martin Doerr; Maria Theodoridou (2017): Report on the commom semantic framework. PARTHENOS deliverable D5.1. Veröffentlicht am 31.04.2017. Verfügbar unter http://www.parthenosproject.eu/Download/Deliverables/D5.1_Common_Semantic_Framework_Appendices.pdf.

Degl'Innocenti, Emiliano; Giacomi, Roberta; Haaf, Susanne; Krauwer, Steven; Illmayer, Klaus; Madonna, Antonio Davide; Sanesi, Maurizio (2018): Report in the assessment of Interoperability, Services and tools. PARTHENOS deliverable D2.4. Veröffentlicht am 26.04.2018. Verfügbar unter http://www.parthenosproject.eu/Download/Deliverables/D2.3_Assessment_Data_Policies_Standardization.pdf.

Drude, Sebastian; Di Giorgio, Sara; Ronzino, Paola; Links, Petra; Van Nispen, Annelies; Verbrugge, Karolien; DegI'Innocenti, Emiliano; Stiller, Juliane; Oltersdorf, Jenny; Spiecker, Claus (2016): Report on user requirements, PARTHENOS deliverable D2.1. Veröffentlicht am 20.10.2016. Verfügbar unter http://www.parthenos-project.eu/Download/Deliverables/D2.1_Userrequirements-report-v2.pdf.

Ďurčo, Matej; Matteo, Lorenzini; Sugimoto, Go (2018): Something will be connected - Semantic mapping from CMDI to Parthenos entities: In: Selected papers from the CLARIN Annual Conference 2017, Budapest, 18-20 September 2017, hg. v. Maciej Piasecki, Linköping Electronic Conference Proceedings, 147, 25-35. Verfügbar unter http://www.ep.liu.se/ecp/contents.asp?issue=147.

Edmond, Jennifer; Garnett, Vicky; Burr, Elisabeth; Laepke, Stefanie; Oltersdorf, Jenny; Goulis, Helen (2016): Initial Training Plan. Veröffentlicht am 07.06.2016. Verfügbar unter http://www.parthenos-project.eu/Download/Deliverables/D7.1_Initial_Training_Plan.pdf.

EOSC Pilot: Shaping the European Open Science Cloud, European Union. Veröffentlicht am 22.11.2017. Verfügbar unter https://issuu.com/eoscpilot/docs/booklet_november2017_web1_22122017.

European Science Foundation (2011): Research Infrastructures in the Digital Humanities (Science Policy Briefing: 42). Verfügbar unter http://archives.esf.org/hosting-experts/scientific-reviewgroups/humanities-hum/strategic-activities/research-infrastructures-in-the-humanities.html.

European Strategy Forum on Research Infrastructures (ESFRI) (2016): Strategy report on research infrastructures: Roadmap 2016, Science and Technology Facilities Council. Verfügbar unter http://www.esfri.eu/sites/default/files/20160309_ROADMAP_browsable.pdf.

Frosini, Luca; Bardi, Alessia; Manghi, Paolo; Pagano, Pasquale (2018): An aggregation framework for Digital Humanities infrastructures: the PARTHENOS experience. In: SCIRES 8 (2018): $133-50$. DOI 10.2423/i22394303v8n1p33. Verfügbar unter http://caspur-ciberpublishing.it/index.php/sciresit/article/view/12906. 


\section{Neuroth}

Dies ist ein Preprint $(\mathrm{cc})$ EY-NC-ND . Die endgültige Publikationsfassung erscheint beim Verlag De Gruyter unter https://www.degruyter.com/view/j/bfup

Hinrichs, Erhard; Thorsten Trippel (2017): CLARIN-D: eine Forschungsinfrastruktur für die sprachbasierte Forschung in den Geistes- und Sozialwissenschaften, In: BIBLIOTHEK - Forschung und Praxis, 41 (1), 45-54.

Hollander, H.S.; Morselli, Francesca; Admiraal, Femmy; Conrad, Anders; Trippel, Thorsten; Zeldenrust, Douwe; Ronzino, Paola; Di Giorgio, Sara; Madonna, Antonio Davide; Hedges, Mark (2017): Report on guidelines for common policies implementation (1). PARTHENOS deliverable D3.1. Veröffentlicht am 25.04.2017. Verfügbar unter http://www.parthenos-project.eu/ Download/Deliverables/D3.1_Guidelines_for_Common_Policies_Implementation.pdf.

Krauwer, Steven; Di Giorgio, Sara; Verbrugge, Karolien; Spiecker, Claus; Chatzimichail, Christos; DegI'Innocenti, Emiliano; Gautier, Hélène; Giacomi, Roberta; Henriksen, Lina; Illmayer, Klaus; Madonna, Antonio Davide; Sanesi, Maurizio (2017). Report on the assessment of data policies and standardization. PARTHENOS Deliverable D2.3. Veröffentlicht am 31.07.2017. Verfügbar unter http://www.parthenosproject.eu/Download/Deliverables/D2.3_Assessment_Data_Policies_Standardization.pdf. Oltersdorf, Jenny; Edmond, Jennifer; Garnett, Vicky; Henriksen, Lina; Mergoupi, Eirini-Savaidou; Povlsen, Claus (2016): Report on the assessment of the education and training plans and activities. PARTHENOS deliverable D2.2. Veröffentlicht am 20.20.2016. Verfügbar unter http://www.parthenosproject.eu/Download/Deliverables/D2.2_Report_Assessment_Education_Training.pdf.

Pagano, Pascquale; Leonardo Candela; Massimiliano Assante; Luca Frossini; Paolo Manghi; Allessia Bardi; Fabio Sinibaldi (2016): PARTHENOS cloud infrastructure. PARTHENOS deliverable D6.1. Verfügbar unter http://www.parthenosproject.eu/Download/Deliverables/D6.1_Parthenos_Cloud_Infrastructure_FINAL.pdf.

PARTHENOS (2017): The FAIR principles and the EOSC concept in the research community of Digital Humanities, Language Studies and Cultural Heritage: An expeditionary survey. Verfügbar unter http://www.parthenos-project.eu/Download/PARTHENOS_FAIR_EOSC_survey.pdf.

Richter, Thomas (2018): Rat für Informationsinfrastrukturen begrüßt nationale Forschungsdateninfrastruktur, Pressemitteilung, 19.11.2018. Verfügbar unter https://idwonline.de/de/news706299.

Romary, Laurent (2017): Report on standardization. PARTHENOS deliverable D4.2. Veröffentlicht am 28.04.2017. Verfügbar unter http://www.parthenosproject.eu/Download/Deliverables/D4.2_Report_Standardization.pdf.

Spiecker, Claus; Oltersdorf, Jenny; Wuttke, Ulrike; Edmond, Jennifer; Garnett. Vicky; Läpke, Stefanie (2017): Report on training and education activities and updated planning. Veröffentlicht am 22.04.2017. Verfügbar unter http://www.parthenosproject.eu/Download/Deliverables/D7.2_Training_Plan_FINAL.pdf.

Spiecker, Claus (2018). Neue Wege wissenschaftlicher Kommunikation - der PARTHENOS Hub (Publikations- und Interaktionsplattform), Abstrakt und Vortragsfolien, 107. Deutscher Bibliothekartag in Berlin, 2018. Verfügbar unter https://opus4.kobv.de/opus4-bibinfo/frontdoor/index/index/docld/3707.

Wilkinson, Mark D.; Dumontier, Michel; Aalbersberg, IJsbrand Jan; Appleton, Gabrielle; Axton, Myles; Baak, Arie; Blomberg, Niklas (2016). The FAIR Guiding Principles for scientific data management and stewardship. Scientific Data. Verfügbar unter https://www.nature.com/articles/sdata201618.

Wuttke, Ulrike (2019, in prep). Evaluation study of the PARTHENOS eHumanities and eHeritage Webinar Series. In Liber Quarterly.

Wuttke, Ulrike; Sierig, Rebecca; Neuroth, Heike (2018). Developing the "PARTHENOS eHumanities and eHeritage Webinar Series": From webinars to online teaching resources. Digital Humanities Benelux 2018, Amsterdam. Verfügbar unter http://2018.dhbenelux.org/wp- 
Preprints der Zeitschrift BIBLIOTHEK - Forschung und Praxis, 2019, AR 324 Wuttke, Spiecker und Neuroth

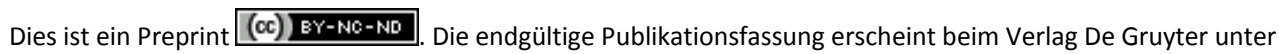
https://www.degruyter.com/view/j/bfup

content/uploads/sites/8/2018/05/DH-Benelux-Submission-PARTHENOS-Webinars-FinalUpload.pdf bzw. https://zenodo.org/record/1257320\#.XBzOL2ICdP8.

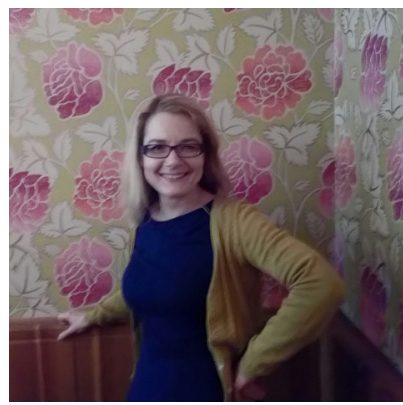

Dr. Ulrike Wuttke

Fachhochschule Potsdam (FHP)

Fachbereich Informationswissenschaften

Kiepenheuer Allee 5

D-14469 Potsdam

wuttke@fh-potsdam.de

http://orcid.org/0000-0002-8217-4025

Claus Spiecker (kein Foto erwünscht!)

Fachhochschule Potsdam (FHP)

Fachbereich Informationswissenschaften

Kiepenheuer Allee 5

D-14469 Potsdam

spiecker@fh-potsdam.de

http://orcid.org/0000-0001-9947-8810

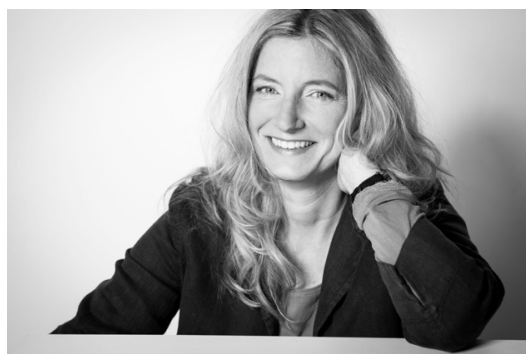

Prof. Dr. rer. nat. Heike Neuroth

Fachhochschule Potsdam (FHP)

Fachbereich Informationswissenschaften

Kiepenheuer Allee 5

D-14469 Potsdam

neuroth@fh-potsdam.de

http://orcid.org/0000-0002-3637-3154 